\title{
ABORDAJE LAPAROSCÓPICO DE UN TUMOR RETROPERITONEAL: REPORTE DE UN CASO Y REVISION DE LA LITERATURA
}

Gonzalo Vitagliano y Carlos Ameri.

Servicio de Urología. Hospital Alemán. Buenos Aires. Argentina.

Resumen.- OBJETIVOS: Los tumores retroperitoneales son infrecuentes y usualmente la mayoría son malignos. Presentamos el abordaje y resolución por vía laparoscópica de un leiomiosarcoma retroperitoneal de bajo grado.

MÉTODOS: Se presenta una paciente de 56 años de edad con diagnóstico de una masa retrorenal de $12 \mathrm{~cm}$ en su diámetro mayor que fue intervenida laparoscopicamente por vía transperitoneal. Durante la cirugía el tumor fue claramente visualizado sobre el aspecto latero-posterior del riñón izquierdo. Utilizando una técnica oncologicamente segura, el tumor fue cuidadosamente liberado de la cápsula renal y extraído en una bolsa plástica por una pequeña incisión en la fosa ilíaca izquierda.

Gonzalo Vitagliano Zabala $26643^{\circ} \mathrm{B}$

Capital Federal. Buenos Aires. Código postal 1426 (Argentina). gonzalovitagliano@hotmail.com

Trabajo recibido: 26 de febrero 2009. 
RESULTADOS: El tiempo operatorio fue de 135 minutos y el sangrado estimado en $200 \mathrm{ml}$. La paciente evolucionó favorablemente y fue externada al tercer día postoperatorio. El análisis final de la pieza operatoria develó un leiomiosarcoma de muy bajo grado de malignidad. Luego de 6 meses de seguimiento, la paciente se encuentra libre de enfermedad y completamente recuperada.

CONCLUSIONES: Aunque la cirugía abierta continua siendo el estándar en el tratamiento de todos los tumores retroperitoneales, el abordaje laparoscópico de una masa retroperitoneal es técnicamente factible y oncologicamente seguro. Se necesitan mayores estudios para probar su efectividad a largo plazo.

Palabras clave: Tumores retroperitoneales. Leiomiosarcoma. Laparoscopía.

Summary.- OBJECTIVES: Retroperitoneal tumors are a rare finding and most masses are of malignant origin. We report laparoscopic management of a low grade retroperitoneal leiomyosarcoma.

METHODS: A 56 year old woman with a left $12 \mathrm{~cm}$ retrorenal mass underwent transperitoneal laparoscopic exploration. The tumor was clearly visualized on the lateral aspect of the left kidney. Following basic oncologic principles, the mass was carefully dissected off the renal capsule and extracted in a laparoscopic bag through a small incision on the left lower quadrant.

RESULTS: Operative time was 135 minutes and estimated surgical bleeding was $200 \mathrm{ml}$. The patient evolved uneventfully and was discharged home on postoperative day 3. Final pathological analysis revealed that the lesion was a very low grade leiomyosarcoma. At six months followup the patient remains disease free and has completely recovered.

CONCLUSIONS: Even though open surgery remains the standard treatment for all retroperitoneal tumors, the laparoscopic approach of a retroperitoneal mass is technically feasible and oncologically safe. Further studies will show long term results.

Keywords: Retroperitoneal tumor. Leiomyosarcoma. Laparoscopy.

\section{INTRODUCCIÓN}

Los tumores retroperitoneales son infrecuentes. Corresponden al $0.07 \%$ a $0.6 \%$ de todos los tumores sólidos del organismo (1). Los tumores benignos son infrecuentes y generalmente la etiología es maligna (1). Los síntomas asociados a las masas retroperitoneales son vagos e inespecíficos y suelen estar correlacionados con el tamaño y relación de la masa con estructuras vecinas. Casi todos los tumores retroperitoneales requieren de cirugía abierta para su efectivo control. No obstante, existen reportes aislados de manejo laparoscópico de schwanomas, teratomas, liposarcomas, linfangiomas, hemangiomas y leiomiosarcomas ubicados en el retroperitoneo $(1,4-9)$. La urolaparoscopía ha evolucionado desde su comienzo y actualmente muchas patologías se benefician de la baja morbilidad de este abordaje quirúrgico. Para realizar una exploración laparoscópica del retroperitoneo se requiere de entrenamiento laparoscópico avanzado. En nuestro centro, la habilidades requeridas se han alcanzado realizando pieloplastías, nefrectomías parciales y radicales, prostatectomías y cistectomías radicales por vía laparoscópica.

Presentamos entonces un caso de un leiomiosarcoma retroperitoneal de bajo grado de malignidad abordado y resuelto por vía laparoscópica.

\section{CASO CLÍNICO}

Una mujer de 56 años fue evaluada inicialmente por desordenes intestinales. El examen físico y la rutina de laboratorio inicial fueron normales. Una ecografía abdominal evidencio una masa sólida de $12 \mathrm{~cm}$ por detrás del riñón izquierdo que presentaba vascularizacion anómala. La tomografía computada mostraba una masa bien circunscripta por detrás del riñón izquierdo que realzaba tras la administración del contraste endovenoso y presentaba un claro plano graso entre el riñón izquierdo y la masa (Figura 1). Se realizó una punción con aguja fina para obtener histología. El reporte anatomopatológico fue de un tumor mesenquimático benigno o de bajo potencial maligno. Se decidió la exploración por vía laparoscópica.

Técnica quirúrgica: Bajo anestesia general el paciente fue posicionado en decúbito lateral derecho luego de la colocación de sonda orogastrica y vesical (Figura 2). Se administro una único dosis de cefalotina $2 \mathrm{gr}$ por via endovenosa. El pneumoperitoneo se realizo con aguja de Veress a $12 \mathrm{mmHg}$. Dos trocares de $12 \mathrm{~mm}$ y $5 \mathrm{~mm}$ respectivamente fueron colocados formando un rombo como se observa en la Figura 3. El colon y el bazo fueron ampliamente movilizados hacia la línea media. Luego de abrir la fascia de Gerota la masa fue claramente visualizada sobre el borde externo del riñón. Preservando los límites tumorales se libero la misma de su contacto con la cápsula renal. Se observo un área de $2 \mathrm{~cm}$ firmemente adherida a la cápsula renal requiriendo de resección de la misma y posterior fulguración del lecho cruento con láser de argón. La pieza quirúrgica fue extraída en una bolsa plástica mediante la prolongación oblicua de la incisión del trocar del flanco izquierdo (Figura 4). Un drenaje tipo Blake fue dejado por el orificio del trocar de $5 \mathrm{~mm}$. El tiempo operatorio fue de 135 minutos con un sangrado estimado en 
$200 \mathrm{ml}$. La paciente evoluciono favorablemente y fue dada de alta al tercer día postoperatorio. El análisis patológico final evidencio un leiomiosarcoma de muy bajo grado de malignidad. Tras un seguimiento de 6 meses la paciente se ha recuperado completamente sin evidencia de su enfermedad de base.

\section{DISCUSIÓN}

Los tumores retroperitoneales de tejidos blandos son de muy baja frecuencia. Se asocian con síntomas vagos e inespecíficos que suelen correlacionarse con la ubicación del tumor y su contacto con estructuras vecinas (2). Los síntomas mas frecuentes incluyen dolor lumbar o abdominal, síntomas genitourinarios, gastrointestinales (nauseas, vómitos y constipación) como también edema genital y de miembros inferiores secundario a obstrucción linfática (4). No obstante, la mayoría de estos tumores son asintomáticos y suelen ser diagnosticados en forma incidental.
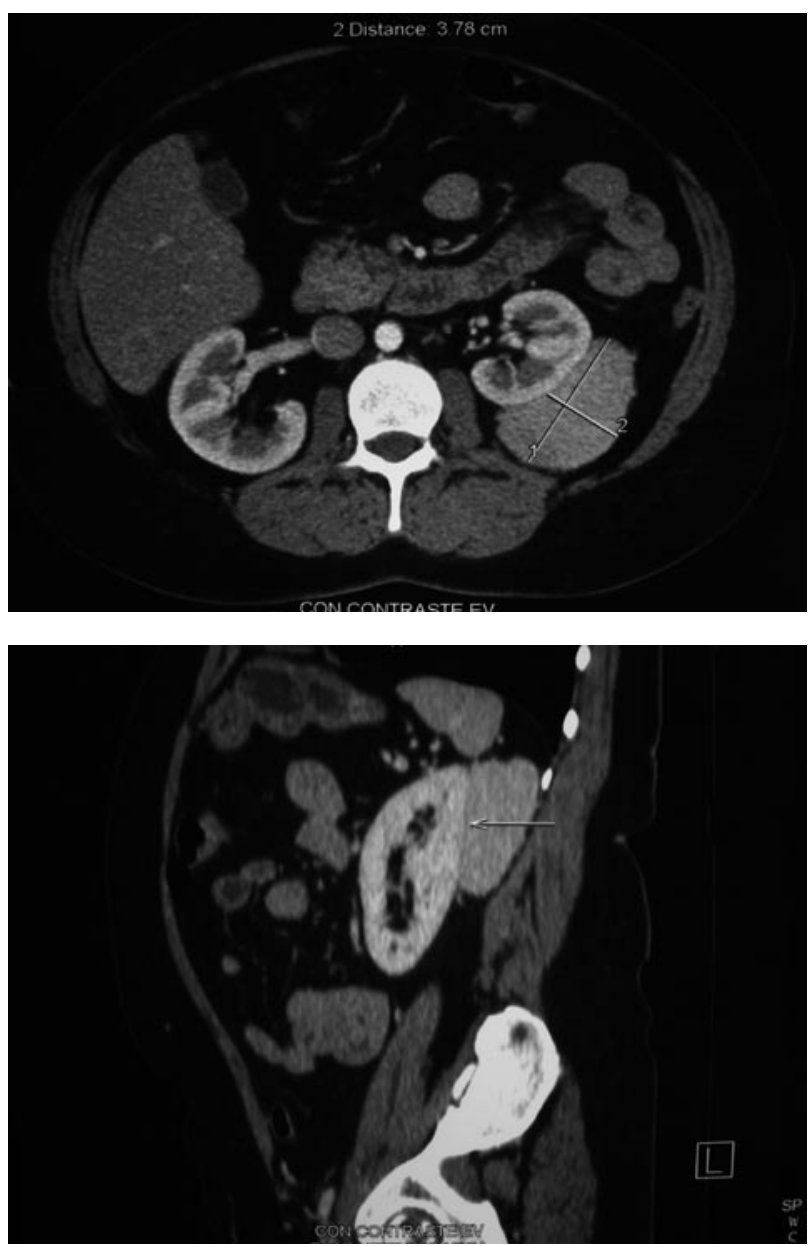

FIGURA 1. Tomografía computada mostrando la masa retrorenal izquierda.
Las ventajas del abordaje laparoscópico sobre la cirugía abierta son bien conocidas. El menor sangrado intraoperatorio, la mejor visualización de las estructuras anatómicas, el menor dolor postoperatorio, la deambulación precoz y la rápida externación hacen de la laparoscopía una buena opción.

El entrenamiento en cirugía laparoscópica permite resolver múltiples escenarios quirúrgicos. Existen reportes de grandes resecciones en tumores renales y suprarrenales. También hay mención de la utilización de esta vía para rescatar un drenaje pancreático y una camisa de Amplatz $(10,11)$.

La cirugía abierta sigue siendo el tratamiento estándar para los tumores retroperitoneales malignos. Es crucial la resección total de la estructura junto con amplios márgenes como también con las estructuras vecinas involucradas para obtener un resultado oncologicaménte seguro. No obstante, recientemente, algunos autores han promulgado la utilización de la laparoscopía para lesiones retroperitoneales malignas (12).

Es imprescindible contar con adecuada imagenología preoperatoria si se pretende realizar un abordaje laparoscópico del retroperitoneo. La tomografía computada es el estándar para estas situaciones. No obstante la RMN es más especifica a la hora de delimitar la lesión y determinar la invasión a estructuras vecinas como también dar luz sobre la posible etiología de la masa retroperitoneal $(2,4)$.

La punción con aguja fina continua siendo controvertida. No obstante brindar mayor información en el preoperatorio, muchos autores señalan la potencial siembra tumoral como un riesgo innecesario dado que el diagnóstico final solo es obtenido mediante la evaluación histológica del espécimen quirúrgico (4).

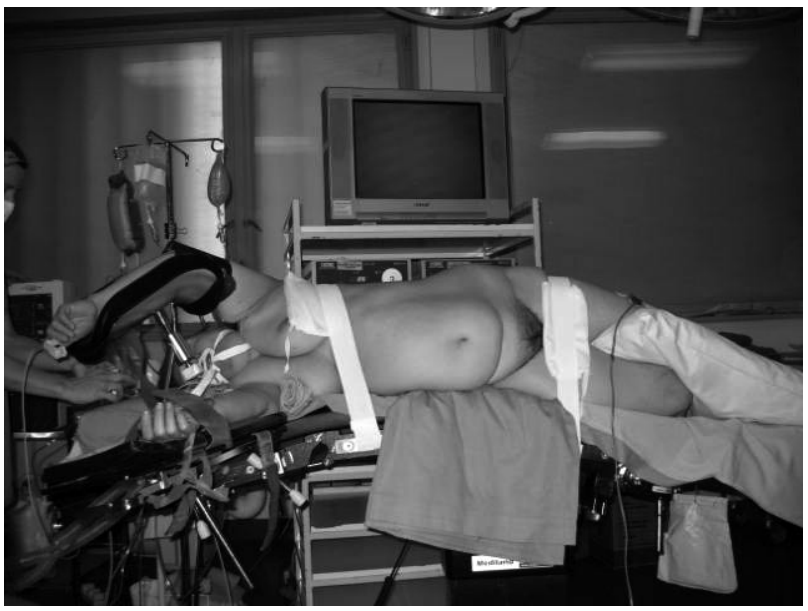

FIGURA 2. Posicionamiento del paciente. 


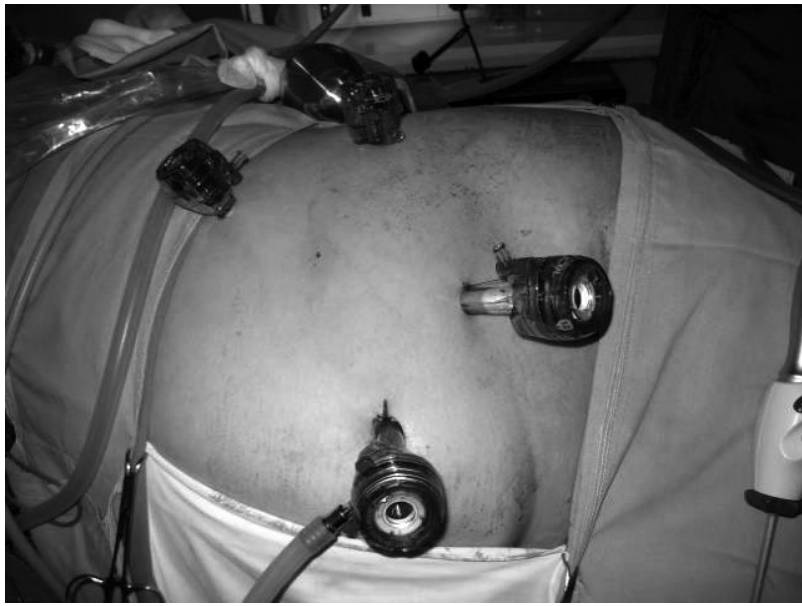

FIGURA 3. Colocación de trocares.

En el paciente reportado la tomografía preoperatorio mostró una masa bien circunscripta con la existencia de buen plano de clivaje con las estructuras vecinas. La punción con aguja fina no evidenciaba malignidad. No se sospechaba que la masa pudiese ser de origen renal. Se realizo un abordaje minimamente invasivo preservando los principios oncológicos. Aunque la anatomía patológica definitiva reporto un leiomiosarcoma de muy bajo grado de malignidad, no fue necesaria una segunda intervención y la paciente se encuentra sin evidencia de su enfermedad.

Este no es el primer reporte de un leiomiosarcoma retroperitoneal abordado por vía laparoscópica, no obstante la literatura es escasa. Uniani et al exitosamente abordaron un leiomiosarcoma retroperitoneal utilizando una técnica laparoscópica sin gas (13). Agresta et al lograron extirpar un leiomiosarcoma de $6 \mathrm{~cm}$ que se encontraba junto al apéndice y al ciego y que inicialmente se considero como un absceso apendicular (9). Dicho paciente fue dado de alta al tercer día postoperatorio sin requerir otro tratamiento $y$ encontrándose libre de enfermedad luego de 18 meses de seguimiento. Los leiomiosarcomas renales son el subtipo mas frecuente de sarcoma renal y en algunos casos pueden ser confundidos con masas retroperitoneales. La célula que los origina es del músculo liso de la cápsula $\circ$ de alguna estructura perinefrica. Como todos los sarcomas, tiende a desplazar mas que a invadir el parénquima y se caracteriza por un crecimiento rápido con metástasis frecuentes y recurrencia local y sistémica (3). Los leiomiomas renales se presentan de manera similar, no obstante el pronostico es favorable (14). Aunque nuestro caso presentaba un área de intimo contacto con la cápsula renal, el análisis histológico posterior y la favorable evolución del caso descartaron la posibilidad de que el tumor fuese de origen renal.

Horiguchi et al reportaron un caso de siembra tumoral tras realizar la reseccion de un liposarcoma mixoide

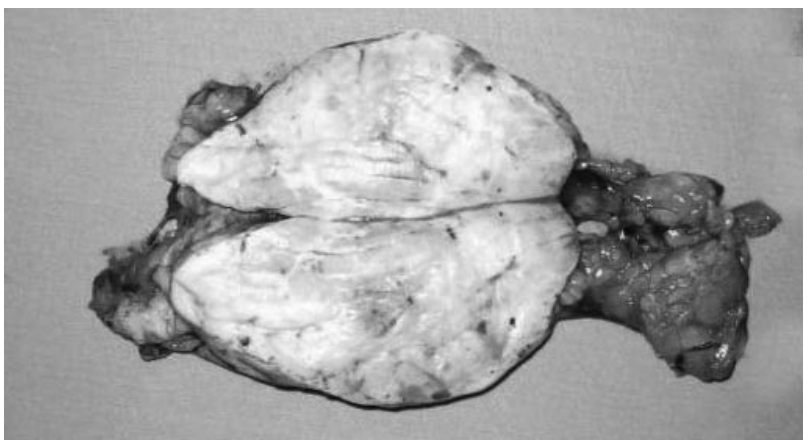

FIGURA 4. Espécimen quirúrgico.

en forma laparoscópica con asistencia manual (15). La diseminación tumoral sigue siendo una preocupación en este tipo de cirugía y todo masa retroperitoneal debe ser tratada inicialmente como maligna. En estos casos la técnica quirúrgica debe ser rigurosa y los criterios oncológicos deben respetarse (16).

\section{CONCLUSIONES}

No se pueden obtener conclusiones definitivas, no obstante es innegable que la laparoscopia ha ganado gran aceptación en la última década y actualmente es considerada una opción valida para el tratamiento de patología maligna. Pese a esto el criterio del cirujano sigue siendo crucial a la hora de indicar un abordaje laparoscópico para una masa retroperitoneal.

\section{BIBLIOGRAFÍA y LECTURAS RECOMENDADAS (*lectura de interés $y^{* *}$ lectura fundamental)}

1. Pinto D, Kaidar-Person O, Cho M, Zundel N, Szomstein S, Rosenthal RJ. Laparoscopic resection of a retroperitoneal degenerative schwannoma: A case report and review of the literature. Surg Laparosc Endosc Percutan Tech. 2008;18(1):121-123.

**2. Cadeddu MO, Mamazza J, Schlachta CM, Seshadri PA, Poulin EC. Laparoscopic excision of retroperitoneal tumors. Surg Laparosc Endosc Percutan Tech. 2008; 18(5):516-519.

*3. Campbell - Walsh Urology 9th edition. 2008.

4. Gatcombe H, Assikis V, Kooby D, Johnstone PA. Primary retroperitoneal teratomas: A review of the literature. J Surg Oncol, 2004;86:107-113.

*5. Dalpiaz O, Gidaro S, Lipsky K, Schips L. Laparoscopic removal of $10 \mathrm{~cm}$ retroperitoneal liposarcoma. Endourol, 2007;21(1):83-84. 
*6. Ball A, Siddiq F, Garcia M, Ganjei-Azar P, Leveillee RJ. Hand-assisted laparoscopic removal of retroperitoneal liposarcoma. Urology, 2005; 65:1226. e9-1226.e11.

7. Tsukamoto T, Tanaka S, Yamamoto T, Kakinoki E, Uemichi A, Kubo S, et al. Laparoscopic excision of a retroperitoneal cystic lymphangioma: Report of a case. Surg Today, 2003; 33:142-144.

8. Perez Martin RN, Estebanez Zarranz J, Velasco Fernandez MdC, Conde Redondo C, Amón Sesmero J, Martinez-Sagarra J. Laparoscopic resection of retroperitoneal venous hemangioma. J Urol, 2004;171:336.

**9. Agresta F, De Simone P, Michelet I, Bedin N. Retroperitoneal leimoyosarcoma mimicking acute appendicitis: Laparoscopic management. JSLS. 2003; 7(2):177-9.

10. Castillo OA, Metrebian E, Vitagliano G. Laparoscopic retrieval of a migrated amplatz sheath. JSLS. 2007;11(1):116-8.

11. Randall J, Mee A, Jones A. Laparoscopic retrieval of a missing pancreatic pseudocyst drain. Surg Laparosc Endosc Percutan Tech, 2008;18(1):92-93.

*12. Moinzadeh A, Gill IS: Laparoscopic radical adrenalectomy for malignancy in 31 patients. J Urol, 2005; 173: 519-25.

13. Uiani MP, Poggi RV, Pinto A, Maruotti RA. Gasless Laparoscopic removal of retroperitoneal leiomyosarcoma. J Laparoendosc Surg, 1995; 5:47-54.

14. Horiguchi A, Saito S, Baba S, Murai M, Mukai M. Port site recurrence after laparoscopic resection of retroperitoneal liposarcoma. J Urol, 1998; 159:1296-1297.

*15. Castillo OA, Vitagliano G. Port site metastasis and tumor seeding in oncologic laparoscopic urology. Urology, 2008; 71(3):372-8. 